Bull. Austral. Math. Soc.

VoL. 57 (1998) [1-24]

\title{
PERTURBED GENERALISED HAMILTONIAN SYSTEMS AND SOME ADVECTION MODELS
}

\author{
Jibin Li, J.R. Christie and K. Gopalsamy
}

\begin{abstract}
In this paper, it is shown that the theory of perturbed generalised Hamiltonian systems provides an effective method for understanding the description of flow patterns of some three-dimensional flows. Firstly, theorems for the persistence of periodic solutions of three-dimensional generalised Hamiltonian systems under perturbation are given by developing Melnikov's method. Then, three different systems of three-dimensional steady fluid flows are discussed and the existence or non-existence of periodic solutions of these systems is proved.
\end{abstract}

\section{INTRODUCTION}

It is well known that in the Lagrangian representation, the path $\mathbf{x}(t)$ of the motion of a fluid particle in a steady incompressible flow in $\mathbb{R}^{3}$ is given by the dynamical system $\dot{\mathbf{x}}=\mathbf{v}(\mathbf{x})$ with $\operatorname{div}(\mathbf{v})=0$, where $\mathbf{v}$ denotes the velocity field. When $\mathbf{v}(\mathbf{x})$ is a nonlinear function of $\mathbf{x}$, the dynamical behaviour of the system may exhibit a variety of behaviour patterns including deterministic chaos and periodic motions. In recent years, a large volume of three-dimensional steady and unsteady flow field data has been produced both experimentally and computationally with the advent of large computers and high-speed data-acquisition systems. This data needs to be interpreted and mathematically understood, but there are some difficulties in the study of threedimensional dynamical systems. For example, even though well known, the dynamics of the Lorenz equations have not been completely understood [13].

In this paper, we show that a description of flow patterns using the theory of perturbed generalised Hamiltonian systems provides a framework and a method for overcoming some of the difficulties associated with three-dimensional flows. As examples, we discuss three systems of three-dimensional steady flows. The results show that our method enables some complicated three-dimensional flow patterns to be described in an intelligible and unambiguous manner, and some numerical results of other authors can be explained.

Received 3rd December, 1996

The first author wishes to thank The Flinders University of South Australia for a Visiting Fellowship which enabled the completion of this research. This research was done while the second author was the holder of a Research Science Cadetship from the Defence Science and Technology Organisation (DSTO), Australia.

Copyright Clearance Centre, Inc. Serial-fee code: 0004-9729/98 \$A2.00+0.00. 
Three-dimensional incompressible flows have a property not possessed by planar flows called "helicity" which indicates the linkage or knottedness of the flow. Holm and Kimura [6] have considered two classes of zero-helicity flows. By using numerical simulations, they gave visualised spatial patterns. The so-called Arter flow [2] and Chandrasekhar flow [5] are respectively given by

$$
\begin{aligned}
& \frac{d x}{d t}=-\sin x \cos y \cos z+b \sin (2 x) \cos (2 z) \\
& \frac{d y}{d t}=-\cos x \sin y \cos z+b \sin (2 y) \cos (2 z) \\
& \frac{d z}{d t}=2 \cos x \cos y \sin z-b(\cos (2 x)+\cos (2 y)) \sin (2 z)
\end{aligned}
$$

and

$$
\begin{aligned}
& \frac{d x}{d t}=-\sin x \cos y \cos z-k^{2} \cos x \sin y \cos z \\
& \frac{d y}{d t}=-\cos x \sin y \cos z+k^{2} \sin x \cos y \cos z \\
& \frac{d z}{d t}=2 \cos x \cos y \sin z .
\end{aligned}
$$

The system $(1.1)_{b}$ is a model for the flow with square planform for the onset of RayleighBénard convection. The system $(1.2)_{k}$ is derived as a lowest-mode linearised solution of the Navier-Stokes equations with buoyancy for a rotating fluid in a periodic cube $\left(T^{3}\right)$ under the Boussinesq approximation. Note that $(1.1)_{b=0}$ coincides with $(1.2)_{k=0}$. However, we shall not consider the Chandrasekhar flow in this paper.

On the other hand, Bajer and Moffatt [3] have studied a class of steady confined Stokes flows. The velocity field is given by

$$
\mathbf{v}(x, y, z)=\mathbf{v}_{1}(x, y, z)+\alpha \mathbf{v}_{2}(x, y, z),
$$

where

$$
\begin{aligned}
& \mathbf{v}_{1}(x, y, z)=\left(-8 x y, 11 x^{2}+3 y^{2}+z^{2}+\beta x z-3,2 y z-\beta x y\right), \\
& \mathbf{v}_{2}(x, y, z)=(z, 0,-x) .
\end{aligned}
$$

The system $(1.3)_{\alpha}$ is called the stretch-twist-fold (STF) flow. This is a particular quadratic flow which was devised to represent STF action in earlier dynamo-theory of magneto-hydrodynamics (see [10] and its references). By using adiabatic invariants, that is, the method of averaging, Bajer and Moffatt [3] discussed some dynamical behaviour of $(1.3)_{\alpha}$ and gave numerical pictures. 
The above systems have not been analysed using rigorous mathematical methods. In addition, Armbruster, Guckenheimer and Holmes [1], Jones and Proctor [7] and Proctor and Jones [12] have independently considered the same equations

$$
\begin{aligned}
& \frac{d s_{1}}{d t}=s_{1} s_{2} \cos \phi+\varepsilon s_{1}\left(\nu_{1}+e_{11} s_{1}^{2}+e_{12} s_{2}^{2}\right) \\
& \frac{d s_{2}}{d t}=-s_{1}^{2} \cos \phi+\varepsilon s_{2}\left(\nu_{2}+e_{21} s_{1}^{2}+e_{22} s_{2}^{2}\right) \\
& \frac{d \phi}{d t}=-\left(2 s_{2}-\frac{s_{1}^{2}}{s_{2}}\right) \sin \phi .
\end{aligned}
$$

They obtained the existence of periodic solutions of $(1.4)_{\varepsilon}$. These periodic solutions correspond to periodic modulated travelling waves in thermal convection. A new treatment for $(1.4)_{\varepsilon}$ will be given below.

This paper is organised as follows. In Section 2, we give theorems concerning the persistence of periodic solutions of three-dimensional generalised Hamiltonian systems under perturbation. In Section 3, the Arter flow is discussed. In Section 4 and Section 5 , we respectively study the STF flow and the system $(1.4)_{\epsilon}$. We show the existence or non-existence of periodic orbits for these three systems.

\section{PERIOdic orbits of PERTURBEd GeNERAlised HAMILTONIAN SYSTEMS}

In this section, we begin by giving a brief description of the theory of perturbed generalised Hamiltonian systems in the three-dimensional case. For the more general theory of generalised Hamiltonian systems, the reader is referred to $[\mathbf{8}, \mathbf{1 1}, \mathbf{1 4}]$.

A three-dimensional perturbed generalised Hamiltonian system studied here is taken to be of the following form:

$$
\frac{d x_{i}}{d t}=\left\{x_{i}, H\right\}(x)+\varepsilon g_{i}(x, t), \quad i=1,2,3,
$$

where $x=\left(x_{1}, x_{2}, x_{3}\right) \in \mathbb{R}^{3},\{\cdot, \cdot\}$ denotes the Poisson bracket $[11]$ and $H \in$ $C^{r}\left(\mathbb{R}^{3}\right), r \geqslant 3$, is called the Hamiltonian function. We assume that each $g_{i}$ is sufficiently smooth $\left(C^{r}, r \geqslant 2\right)$. We let

$$
f_{i}(x)=\left\{x_{i}, H\right\}(x)=\sum_{j=1}^{3} J_{i j}(x) \frac{\partial H}{\partial x_{j}}(x), \quad i=1,2,3,
$$

where $J_{i j}(x)=\left\{x_{i}, x_{j}\right\}$. The $3 \times 3$ anti-symmetric matrix $J(x)=\left(J_{i j}(x)\right)$ is called the structure matrix of a generalised Hamiltonian system. We note that the system $(2.1)_{\varepsilon}$ can be rewritten in the form

$$
\frac{d x}{d t}=J(x) \nabla H(x)+\varepsilon g(x, t),
$$


where $g=\left(g_{1}, g_{2}, g_{3}\right)$. Suppose that the perturbation terms $g_{i}(x, t), i=1,2,3$, are $T$-periodic with respect to $t$, and $0 \leqslant \varepsilon \ll 1$.

Definition 2.1: Let $P$ be a Poisson manifold. A smooth, real-valued function $C: P \rightarrow \mathbb{R}$ is called a Casimir function if $\{C, F\}=0$ for all functions $F: P \rightarrow \mathbb{R}$.

Note that a Poisson manifold is a manifold with a Poisson bracket. We make the following assumptions about the unperturbed system $(2.1)_{\varepsilon=0}$ :

$\left(\mathrm{A}_{1}\right)$ We assume that there exists a Casimir function $C$ of the Poisson manifold $\left(\mathbb{R}^{3},\{\cdot, \cdot\}\right)$, which is defined on the regular point set $M=\left\{x \in \mathbb{R}^{3}\right.$ : rank $(J(x))=2\}$ (or a connected open subset $U \subset M$ ), such that the gradient $\nabla C(x) \neq 0$ for each $x \in M_{c}=\{x \in M: C(x)=c\}$ with constant $c$ satisfying $|c| \leqslant \delta$ for some $\delta>0$ (that is, $M_{c}$ contains no critical points of $C$ ).

$\left(\mathrm{A}_{2}\right)$ In addition to assumption $\mathrm{A}_{1}$, we assume that for each value of $c$ in some open interval $I \subset\{c \in \mathbb{R}:|c| \leqslant \delta\}$, on the level set $M_{c}$ the unperturbed system $(2.1)_{\varepsilon=0}$ possesses a one parameter family of periodic orbits, $q_{0}^{\alpha}(t-\theta, c), \alpha \in L(c)$, where $L(c) \subset \mathbb{R}$ is an open interval and $\theta$ denotes the "phase" or starting point of the orbit. We denote the period of $q_{0}^{\alpha}(t-\theta, c)$ by $T(\alpha, c)$.

Definition 2.2: Let $P$ be a Poisson manifold, let $C: P \rightarrow \mathbb{R}$ be a Casimir function and let $c \in \mathbb{R}$. Then, the set $P_{c}=\{x \in P: C(x)=c\}$ is called a symplectic leaf of $C$ on $P$.

Evidently, the set $M$ is a Poisson manifold with two-dimensional symplectic leaves $M_{c}$ of $C$, and each leaf $M_{c}$ is an invariant manifold of the flow defined by the unperturbed system $(2.1)_{\varepsilon=0}$.

Under the above assumptions, we study the existence of periodic orbits of the perturbed system $(2.1)_{\varepsilon}$. Firstly, the following perturbation results will be useful. Consider a subset of the two parameter family of periodic orbits of $(2.1)_{\varepsilon=0}$ whose period is uniformly bounded above. Let $\widehat{L}(c) \subset L(c)$ denote the set of $\alpha$ such that on the level set $M_{c}$ the periods $T(\alpha, c)$ of the periodic orbits are uniformly bounded above, say by a constant $K$.

THEOREM 2.1. Let $q_{0}^{\alpha}(t-\theta, c)$ be a periodic orbit of the unperturbed system $(2.1)_{\varepsilon=0}$ with period $T(\alpha, c)<K$. Then, there exists a perturbed orbit $q_{\varepsilon}^{\alpha}(t, \theta, c)$, not necessarily periodic, which can be expressed as

$$
q_{\varepsilon}^{\alpha}(t, \theta, c)=q_{0}^{\alpha}(t-\theta, c)+\varepsilon q_{1}^{\alpha}(t, \theta, c)+\mathcal{O}\left(\varepsilon^{2}\right)
$$

uniformly in $t \in[\theta, \theta+T(\alpha, c)]$ for $\varepsilon$ sufficiently small and all $\alpha \in \widehat{L}(c)$. 
REMARK 2.1. This result follows directly from regular perturbation theory and Gronwall estimates (see [15]). The restriction to $\widehat{L}(c)$ avoids problems that arise when periodic orbits limit on homoclinic orbits and the period becomes unbounded. Furthermore, $q_{1}^{\alpha}(t, \theta, c)$ may be found by solving the variational equation

$$
\frac{d}{d t}\left(q_{1}^{\alpha}(t, \theta, c)\right)=D f\left(q_{0}^{\alpha}(t-\theta, c)\right) q_{1}^{\alpha}(t, \theta, c)+g\left(q_{0}^{\alpha}(t-\theta, c), t\right),
$$

where $D f$ denotes the Jacobi matrix of $f$.

We want to determine if any of the two parameter family of periodic orbits in the unperturbed system persist as periodic orbits in the perturbed system. To do this, we first suspend the perturbed system $(2.1)_{\varepsilon}$ by rewriting it as an autonomous system of differential equations where we denote $f=\left(f_{1}, f_{2}, f_{3}\right)$ and $g=\left(g_{1}, g_{2}, g_{3}\right)$. We define the function $\Phi(t)=t(\bmod T)$; by the $T$-periociicity of $g$ we then have

$$
\left.\begin{array}{l}
\frac{d x}{d t}=f(x)+\varepsilon g(x, \Phi) \\
\frac{d \Phi}{d t}=1
\end{array}\right\}, \quad(x, \Phi) \in \mathbb{R}^{3} \times S_{T},
$$

where $S_{T}=\mathbb{R}(\bmod T)$. We remark that this suspension makes sense even when $g$ is independent of $\Phi$, although it then becomes trivial. Secondly, we shall reduce the study of the four-dimensional problem $(2.4)_{\varepsilon}$ to a three-dimensional Poincaré map. Define a global cross-section transverse to the vector field $(2.4)_{\varepsilon}$ by

$$
\Sigma^{0}=\left\{(x, \Phi) \in \mathbb{R}^{3} \times S_{T}: \Phi=0(\bmod T)\right\}
$$

and define the Poincaré map associated with the system $(2.4)_{\varepsilon}, P_{\varepsilon}: \Sigma^{0} \rightarrow \Sigma^{0}$, by

$$
P_{\varepsilon}: x_{\varepsilon}(0) \rightarrow x_{\varepsilon}(T),
$$

where $\left(x_{\varepsilon}(t), \Phi(t)\right)$ denotes the solution of the system $(2.4)_{\varepsilon}$ starting from the point $\left(x_{\varepsilon}(0), 0\right) \in \Sigma^{0}$. The $m$ th iterate of the Poincaré map, $P_{\varepsilon}^{m}$, is

$$
P_{\varepsilon}^{m}: x_{\varepsilon}(0) \rightarrow x_{\varepsilon}(m T)
$$

Thus, the study of subharmonic periodic orbits of $(2.4)_{\epsilon}$ is reduced to the study of fixed points of the map $P_{\varepsilon}^{m}$. In the following, we shall explicitly construct an applicable expression for $P_{\varepsilon}^{m}$ (in a new coordinate system) from the perturbed vector field (2.4) $)_{\varepsilon}$; the structure of the unperturbed phase space will form the framework for the analysis of the problem. We shall restrict ourselves to a region where the periods of the unperturbed periodic orbits are uniformly bounded above by a constant $K$ (so Theorem 2.1 applies). 
From Darboux' Theorem (see [11]) and the existence theorem of the action-angle variables of a generalised Hamiltonian system in [8], we note that in a neighbourhood of an unperturbed periodic orbit there exists a coordinate transformation of the form

$$
x=\left(x_{1}, x_{2}, x_{3}\right) \rightarrow(a(x), \phi(x)(\bmod 2 \pi), C(x))
$$

such that in the new coordinates, the unperturbed Hamiltonian is only dependent on $a$ and $c$, that is, $H(x)=\widehat{H}(a, c)$, and elements of the structure matrix of the Poisson bracket become

$$
\{a, \phi\}=-1, \quad\{a, c\}=0, \quad\{\phi, c\}=0,
$$

where $C(x)=c$ is the Casimir function given in assumption $\mathrm{A}_{1}$ and $\{\cdot, \cdot\}$ is the Poisson bracket in $\mathrm{A}_{1}$. Under the transformation, $(2.4)_{\varepsilon}$ becomes

$(2.9)_{\varepsilon}$

$$
\begin{aligned}
\frac{d a}{d t} & =\{a, \widehat{H}(a, c)\}+\varepsilon\langle\nabla a, g\rangle(a, \phi, c, \Phi) \\
\frac{d \phi}{d t} & =\{\phi, \widehat{H}(a, c)\}+\varepsilon\langle\nabla \phi, g\rangle(a, \phi, c, \Phi) \\
\frac{d c}{d t} & =\{c, \widehat{H}(a, c)\}+\varepsilon\langle\nabla C, g\rangle(a, \phi, c, \Phi) \\
\frac{d \Phi}{d t} & =1
\end{aligned}
$$

where $\langle\cdot, \cdot\rangle$ denotes the inner product of vectors and $\nabla$ denotes the gradient operator. It follows from (2.8) and the definition of Poisson bracket that

$$
\{a, \widehat{H}(a, c)\}=\{c, \widehat{H}(a, c)\}=0, \quad\{\phi, \widehat{H}(a, c)\}=\left.\frac{\partial \widehat{H}}{\partial a}\right|_{\mathbf{c}}
$$

where $\left.\frac{\partial \widehat{H}}{\partial a}\right|_{c}$ means the partial derivative of $\widehat{H}$ with respect to $a$ with $c$ held fixed, so the system $(2.9)_{\varepsilon}$ can be further reduced to

$$
\begin{aligned}
\frac{d a}{d t} & =\varepsilon\langle\nabla a, g\rangle(a, \phi, c, \Phi) \equiv \varepsilon F(a, \phi, c, \Phi) \\
\frac{d \phi}{d t} & =\left.\frac{\partial \widehat{H}}{\partial a}\right|_{c}+\varepsilon\langle\nabla \phi, g\rangle(a, \phi, c, \Phi) \equiv \Omega(a, c)+\varepsilon G(a, \phi, c, \Phi) \\
\frac{d c}{d t} & =\varepsilon\langle\nabla C, g\rangle(a, \phi, c, \Phi) \equiv \varepsilon R(a, \phi, c, \Phi) \\
\frac{d \Phi}{d t} & =1
\end{aligned}
$$


where $(a, \phi, c, \Phi) \in A \times S_{2 \pi} \times I \times S_{T}$, in which $A \subset \mathbb{R}^{+}$is a finite interval and $I$ is defined in assumption $\mathrm{A}_{2} . F, G$ and $R$ are $T$-periodic in $\Phi$ and $\Omega(a, c)=\left.\frac{\partial \hat{H}}{\partial a}\right|_{c}$ is the angular frequency of the closed orbit in the unperturbed system on the $c=$ constant symplectic leaf with action $a$ and energy $\widehat{H}(a, c)$. We can easily solve the unperturbed system $(2.11)_{\varepsilon=0}$ (without loss of generality, take the initial value $\Phi(0)=0$ ) and obtain the solution

$$
a=a_{0}, \quad \phi=\Omega\left(a_{0}, c_{0}\right) t+\phi_{0}(\bmod 2 \pi), \quad c=c_{0}, \quad \Phi=t(\bmod T) .
$$

Thus, the coordinate $a$ plays the role of the parameter $\alpha$ in assumption $\mathrm{A}_{2}$.

Now we construct an approximation to the Poincaré map associated with system $(2.11)_{\varepsilon}$. The cross-section to the flow defined by $(2.11)_{\varepsilon}$ is

$$
\widehat{\Sigma}^{0}=\left\{(a, \phi, c, \Phi) \in A \times S_{2 \pi} \times I \times S_{T}: \Phi=0(\bmod T)\right\}
$$

and the $m$ th iterate of the Poincare map, $\widehat{P}_{\varepsilon}^{m}$, in the new coordinates is

$$
\widehat{P}_{\varepsilon}^{m}:\left(a_{\varepsilon}(0), \phi_{\varepsilon}(0), c_{\varepsilon}(0)\right) \rightarrow\left(a_{\varepsilon}(m T), \phi_{\varepsilon}(m T), c_{\varepsilon}(m T)\right)
$$

where $\left(a_{\varepsilon}(t), \phi_{\varepsilon}(t), c_{\varepsilon}(t), \Phi(t)\right)$ is the solution of the system $(2.11)_{\varepsilon}$ starting from $\left(a_{0}, \phi_{0}, c_{0}, 0\right) \in \widehat{\Sigma}^{0}$. Using Theorem 2.1 and the solution to the unperturbed system given by $(2.12)$, we can approximate the Poincaré map using regular perturbation theory (note that we define $\widehat{T}(a, c)=T(\alpha, c)$ )

$$
\begin{aligned}
& a_{\varepsilon}(t)=a_{0}+\varepsilon a_{1}(t)+\mathcal{O}\left(\varepsilon^{2}\right) \\
& \phi_{\varepsilon}(t)=\Omega\left(a_{0}, c_{0}\right) t+\phi_{0}+\varepsilon \phi_{1}(t)+\mathcal{O}\left(\varepsilon^{2}\right) \\
& c_{\varepsilon}(t)=c_{0}+\varepsilon c_{1}(t)+\mathcal{O}\left(\varepsilon^{2}\right)
\end{aligned}
$$

where $t \in\left[0, \widehat{T}\left(a_{0}, c_{0}\right)\right]$ in which $\widehat{T}\left(a_{0}, c_{0}\right)=(2 \pi) / \Omega\left(a_{0}, c_{0}\right)$, and $a_{1}, \phi_{1}$ and $c_{1}$ satisfy the variational system

$$
\begin{aligned}
\frac{d a_{1}}{d t} & =F\left(a_{0}, \Omega\left(a_{0}, c_{0}\right) t+\phi_{0}, c_{0}, t\right) \\
\frac{d \phi_{1}}{d t} & =\frac{\partial \Omega}{\partial a}\left(a_{0}, c_{0}\right) a_{1}(t)+\frac{\partial \Omega}{\partial c}\left(a_{0}, c_{0}\right) c_{1}(t)+G\left(a_{0}, \Omega\left(a_{0}, c_{0}\right) t+\phi_{0}, c_{0}, t\right) \\
\frac{d c_{1}}{d t} & =R\left(a_{0}, \Omega\left(a_{0}, c_{0}\right) t+\phi_{0}, c_{0}, t\right)
\end{aligned}
$$


and hence (choosing $a_{1}(0)=\phi_{1}(0)=c_{1}(0)=0$ )

$$
\begin{aligned}
a_{1}(m T)= & \int_{0}^{m T} F\left(a_{0}, \Omega\left(a_{0}, c_{0}\right) t+\phi_{0}, c_{0}, t\right) d t \equiv \bar{M}_{1}^{m / n}\left(a_{0}, \phi_{0}, c_{0}\right) \\
\phi_{1}(m T)= & \frac{\partial \Omega}{\partial a}\left(a_{0}, c_{0}\right) \int_{0}^{m T} \int_{0}^{t} F\left(a_{0}, \Omega\left(a_{0}, c_{0}\right) \eta+\phi_{0}, c_{0}, \eta\right) d \eta d t \\
& +\frac{\partial \Omega}{\partial c}\left(a_{0}, c_{0}\right) \int_{0}^{m T} \int_{0}^{t} R\left(a_{0}, \Omega\left(a_{0}, c_{0}\right) \eta+\phi_{0}, c_{0}, \eta\right) d \eta d t \\
& +\int_{0}^{m T} G\left(a_{0}, \Omega\left(a_{0}, c_{0}\right) t+\phi_{0}, c_{0}, t\right) d t \equiv \bar{M}_{2}^{m / n}\left(a_{0}, \phi_{0}, c_{0}\right) \\
c_{1}(m T)= & \int_{0}^{m T} R\left(a_{0}, \Omega\left(a_{0}, c_{0}\right) t+\phi_{0}, c_{0}, t\right) d t \equiv \bar{M}_{3}^{m / n}\left(a_{0}, \phi_{0}, c_{0}\right)
\end{aligned}
$$

Recall that the approximation (2.15) is uniformly valid for one period of an unperturbed orbit, $\widehat{T}(a, c)=m T$. For the case of ultrasubharmonics, $\widehat{T}(a, c)=(m T / n), n \geqslant 2$, where $m$ and $n$ are relatively prime, and the approximation is not uniformly valid since $\varepsilon$ must shrink to zero as $n$ increases. We define the vector $\bar{M}^{m / n}$ as

$$
\bar{M}^{m / n}\left(a_{0}, \phi_{0}, c_{0}\right)=\left(\bar{M}_{1}^{m / n}\left(a_{0}, \phi_{0}, c_{0}\right), \bar{M}_{2}^{m / n}\left(a_{0}, \phi_{0}, c_{0}\right), \bar{M}_{3}^{m / n}\left(a_{0}, \phi_{0}, c_{0}\right)\right)
$$

which we call the subharmonic Melnikov vector. We remark that the superscript $m / n$ denotes our search for periodic orbits which satisfy the resonance relation $\widehat{T}(a, c)=$ $(m T / n)$, where $m$ and $n$ are relatively prime positive integers. In calculations, we often take $n=1$.

According to the definition of $\widehat{P}_{\varepsilon}^{m}$, a fixed point of $\widehat{P}_{\varepsilon}^{m}$ corresponds to a subharmonic orbit of order $m$ of the perturbed system $(2.1)_{\varepsilon}$. Hence, by using $(2.14),(2.15)$ and (2.17), we can deduce the following theorem:

TheOREM 2.2. Suppose $\left(a_{0}, \phi_{0}, c_{0}\right)$ is a point where $\widehat{T}\left(a_{0}, c_{0}\right)=(m T / n)$ and one of the following two groups of conditions is satisfied:

$$
\begin{aligned}
& \text { (I) } \frac{\partial \Omega}{\partial a}\left(a_{0}, c_{0}\right) \neq 0 \text { or } \frac{\partial \Omega}{\partial c}\left(a_{0}, c_{0}\right) \neq 0 \\
& \text { and } \bar{M}_{1}^{m / n}\left(a_{0}, \phi_{0}, c_{0}\right)=\bar{M}_{3}^{m / n}\left(a_{0}, \phi_{0}, c_{0}\right)=0 \\
& \text { and }\left[\frac{\partial \Omega}{\partial a}\left(\frac{\partial \bar{M}_{1}^{m / n}}{\partial \phi} \frac{\partial \bar{M}_{3}^{m / n}}{\partial c}-\frac{\partial \bar{M}_{1}^{m / n}}{\partial c} \frac{\partial \bar{M}_{3}^{m / n}}{\partial \phi}\right)\right. \\
& \left.+\frac{\partial \Omega}{\partial c}\left(\frac{\partial \bar{M}_{1}^{m / n}}{\partial a} \frac{\partial \bar{M}_{3}^{m / n}}{\partial \phi}-\frac{\partial \bar{M}_{1}^{m / n}}{\partial \phi} \frac{\partial \bar{M}_{3}^{m / n}}{\partial a}\right)\right]\left.\right|_{\left(a_{0}, \phi_{0}, c_{0}\right)} \neq 0 \\
& \text { (II) } \frac{\partial \Omega}{\partial a}\left(a_{0}, c_{0}\right)=\frac{\partial \Omega}{\partial c}\left(a_{0}, c_{0}\right)=0
\end{aligned}
$$




$$
\begin{aligned}
& \text { and } \bar{M}_{1}^{m / n}\left(a_{0}, \phi_{0}, c_{0}\right)=\bar{M}_{2}^{m / n}\left(a_{0}, \phi_{0}, c_{0}\right)=\bar{M}_{3}^{m / n}\left(a_{0}, \phi_{0}, c_{0}\right)=0 \\
& \text { and }\left.\frac{\partial\left(\bar{M}_{1}^{m / n}, \bar{M}_{2}^{m / n}, \bar{M}_{3}^{m / n}\right)}{\partial(a, \phi, c)}\right|_{\left(a_{0}, \phi_{0}, c_{0}\right)} \neq 0 .
\end{aligned}
$$

Then, for $\varepsilon$ sufficiently small, $0<\varepsilon \leqslant \varepsilon(n)$, the $m$ th iterate of the Poincaré map $\widehat{P}_{\varepsilon}^{m}$ in (2.14) has a fixed point near the point $\left(a_{0}, \phi_{0}, c_{0}\right)$; thus the corresponding perturbed generalised Hamiltonian system $(2.1)_{\varepsilon}$ has a subharmonic orbit of order $m$. If $n=1$, the result is uniformly valid in $0<\varepsilon \leqslant \varepsilon(1)$.

The proof of Theorem 2.2 is analogous to that in [15, Theorem 3.1]; we therefore omit it. We remark that in the case of (I), no knowledge of $\bar{M}_{2}^{m / n}$ is needed. Thus, evaluation of the double integrals in $\bar{M}_{2}^{m / n}$ is unnecessary in this case.

Theorem 2.2 does not apply in the case of autonomous vector fields. In such cases, it suffices to study a diffeomorphism of $\mathbb{R}^{2}$ obtained by fixing $\phi=\phi_{0}$ and allowing the $a$ and $c$ variables with initial values at $\phi=\phi_{0}$ to evolve in time until they return to $\phi=\phi_{0}$. We then have the following theorem:

THEOREM 2.3. Suppose there exists a point $\left(a_{0}, c_{0}\right)$ such that $\bar{M}_{1}\left(a_{0}, c_{0}\right)=\bar{M}_{3}\left(a_{0}, c_{0}\right)=0$ and $\left.\frac{\partial\left(\bar{M}_{1}, \bar{M}_{3}\right)}{\partial(a, c)}\right|_{\left(a_{0}, c_{0}\right)} \neq 0$. Then, the two-dimensional Poincaré map has an isolated fixed point $\left(a_{0}, c_{0}\right)+\mathcal{O}(\varepsilon)$ which corresponds to an isolated periodic orbit for the three-dimensional flow.

REMARK 2.2. $\bar{M}_{1}$ and $\bar{M}_{3}$ in Theorem 2.3 are defined as in the non-autonomous case except that the limits of integration now become 0 and $\widehat{T}(a, c)$; therefore, we drop the superscript $m / n$. The proof of Theorem 2.3 is similar to that of Theorem 2.2; see [15, Theorem 3.2].

We next discuss whether we can calculate the components of the subharmonic Melnikov vector in (2.17) in the original coordinates. From (2.17), we know that $\bar{M}_{i}^{m / n}(i=1,2,3)$ actually involves the integral of the functions $F, G$ and $R$ along the unperturbed orbit $(a, \Omega(a, c) t+\phi, c)$. If the orbit corresponds to $q_{0}^{\alpha}(t-\theta, c)$ defined in assumption $\mathrm{A}_{2}$, then we have

$$
\begin{aligned}
\bar{M}_{3}^{m / n}(a, \phi, c) & =\int_{0}^{m T} R(a, \Omega(a, c) t+\phi, c, t) d t \\
& =\int_{0}^{m T}\langle\nabla C, g\rangle\left(q_{0}^{\alpha}(t, c), t+\theta\right) d t \equiv M_{3}^{m / n}(\alpha, \theta, c) .
\end{aligned}
$$

Here, $C$ is the Casimir function given in assumption $A_{1}$. Hence the calculation of $\bar{M}_{3}^{m / n}$ can be replaced by the one of $M_{3}^{m / n}$. We note that $\alpha$ plays the same role as $a$ 
does. Along the unperturbed orbit, $\phi$ and $\theta$ satisfy $\phi=\Omega(a, c) \theta$. Similarly, we have

$$
\begin{aligned}
\bar{M}_{1}^{m / n}(a, \phi, c) & =\int_{0}^{m T} F(a, \Omega(a, c) t+\phi, c, t) d t \\
& =\int_{0}^{m T}\langle\nabla a, g\rangle\left(q_{0}^{\alpha}(t, c), t+\theta\right) d t \equiv M_{1}^{m / n}(\alpha, \theta, c) .
\end{aligned}
$$

From the choice of coordinates $(a, \phi, c)$, using these new coordinates we have rewritten the Hamiltonian $H$ as

$$
H(x)=\widehat{H}(a, c)
$$

and in the domain of the new coordinates,

$$
\left.\frac{\partial \widehat{H}}{\partial a}\right|_{c}=\Omega(a, c) \neq 0 .
$$

Thus by the inverse function theorem, we can invert (2.20) to obtain

$$
a=a(\widehat{H}, c) .
$$

Now $H$ and $C$ are functions of $x$, so it immediately follows from the chain rule that

$$
\nabla a(x)=\left.\frac{\partial a}{\partial \widehat{H}}\right|_{c} \nabla H(x)+\left.\frac{\partial a}{\partial c}\right|_{\widehat{H}} \nabla C(x) .
$$

On the other hand, along an unperturbed periodic orbit, the coordinate $a$ is invariant, so differentiating (2.22) with respect to $c$ along this orbit we get

$$
0=\left.\left.\frac{\partial a}{\partial \widehat{H}}\right|_{c} \frac{\partial \widehat{H}}{\partial c}\right|_{a}+\left.\frac{\partial a}{\partial c}\right|_{\widehat{H}}
$$

and hence

$$
\left.\frac{\partial a}{\partial c}\right|_{\widehat{H}}=-\left.\left.\frac{\partial a}{\partial \widehat{H}}\right|_{c} \frac{\partial \widehat{H}}{\partial c}\right|_{a} .
$$

Using (2.21), (2.23) and (2.25), (2.19) becomes

$$
\begin{array}{r}
\int_{0}^{m T}\langle\nabla a, g\rangle\left(q_{0}^{\alpha}(t, c), t+\theta\right) d t=\frac{1}{\Omega(a, c)}\left[\int_{0}^{m T}\langle\nabla H, g\rangle\left(q_{0}^{\alpha}(t, c), t+\theta\right) d t\right. \\
\left.-\left.\frac{\partial \widehat{H}}{\partial c}\right|_{a} \int_{0}^{m T}\langle\nabla C, g\rangle\left(q_{0}^{\alpha}(t, c), t+\theta\right) d t\right]
\end{array}
$$


and since $\Omega(a, c)=(2 \pi) / T(\alpha, c)$, where $T(\alpha, c)$ is the period of $q_{0}^{\alpha}(t, c)$, and using the fact that

$$
\left.\frac{\partial \widehat{H}}{\partial c}\right|_{a}=\frac{\partial \widehat{H}}{\partial c}\left(q_{0}^{\alpha}(0, c)\right),
$$

we obtain the final formula for $M_{1}^{m / n}(\alpha, \theta, c)$ :

$$
\begin{aligned}
M_{1}^{m / n}(\alpha, \theta, c)=\frac{T(\alpha, c)}{2 \pi}\left[\int_{0}^{m T}\right. & \langle\nabla H, g\rangle\left(q_{0}^{\alpha}(t, c), t+\theta\right) d t \\
& \left.\quad-\frac{\partial \widehat{H}}{\partial c}\left(q_{0}^{\alpha}(0, c)\right) \int_{0}^{m T}\langle\nabla C, g\rangle\left(q_{0}^{\alpha}(t, c), t+\theta\right) d t\right]
\end{aligned}
$$

For $\bar{M}_{2}^{m / n}$, we have not found analogously simple transformations which transform it in the coordinates $(a, \phi, c)$ into the original coordinates $x=\left(x_{1}, x_{2}, x_{3}\right)$. It appears that in this case the new coordinates transformation must be explicitly computed. However, by carefully observing Theorem 2.2 and Theorem 2.3 , we see that in case (I) of Theorem 2.2 and in Theorem 2.3, no knowledge of $\bar{M}_{2}^{m / n}$ (or $\bar{M}_{2}$ for Theorem 2.3) is needed, while in case (II) of Theorem $2.2,\left.\frac{\partial \Omega}{\partial c}\right|_{a}=\left.\frac{\partial \Omega}{\partial a}\right|_{c}=0$ mostly occurs when the unperturbed system is linear, and for such systems we generally can explicitly find out the coordinate transformation in (2.7).

On the problems of stability and bifurcation, we can obtain the same results as Wiggins and Holmes [15]; for detailed information, the reader can refer to this reference.

REMARK 2.3. The results of this section can be thought of as generalisations of Wiggins and Holmes [15]; in fact, the system they considered can be regarded as a special case of the perturbed generalised Hamiltonian system. They deal with the situation where the symplectic leaves are horizontal planes. By taking a particular case of the generalised Hamiltonian system, we can make $(2.1)_{\epsilon}$ the system studied by them.

REMARK 2.4. In some cases, the reduced unperturbed two-dimensional system is not Hamiltonian but is integrable. To calculate the Melnikov integral $M_{1}^{m / n}(\alpha, \theta, c)$, it is similar to the original paper of Melnikov [9]. We need to put a factor $\exp \left(-\int_{0}^{t}\right.$ trace $\left.D f\left(q_{0}^{\alpha}(s, c)\right) d s\right)$ into the integrand of $(2.28)$ provided that $\frac{\partial \widehat{H}}{\partial c}\left(q_{0}^{\alpha}(0, c)\right)=0$. In Section 4 and Section 5, we must use this remark.

\section{Arter Flow}

We consider the system $(1.1)_{b}$. It is easy to see that by identifying the planes $x=-\pi$ and $x=\pi, y=-\pi$ and $y=\pi$, and $z=-\pi$ and $z=\pi$, the system (1.1) ${ }_{b}$ can 
be seen as a system defined on a three-dimensional torus $T^{3}$. The planes $x=0, \pm \pi$, $y=0, \pm \pi, z=0, \pm \pi, y=x$ and $y=-x$ are invariant planes of $(1.1)_{b}$, for all $b \in \mathbb{R}$. Therefore, the three-dimensional flow of $(1.1)_{b}$ is preserved in the fundamental cubical cell composed by eight triangular prisms (see Figure 3.1). We only need to investigate the dynamical behaviour of $(1.1)_{b}$ in a triangular prism.

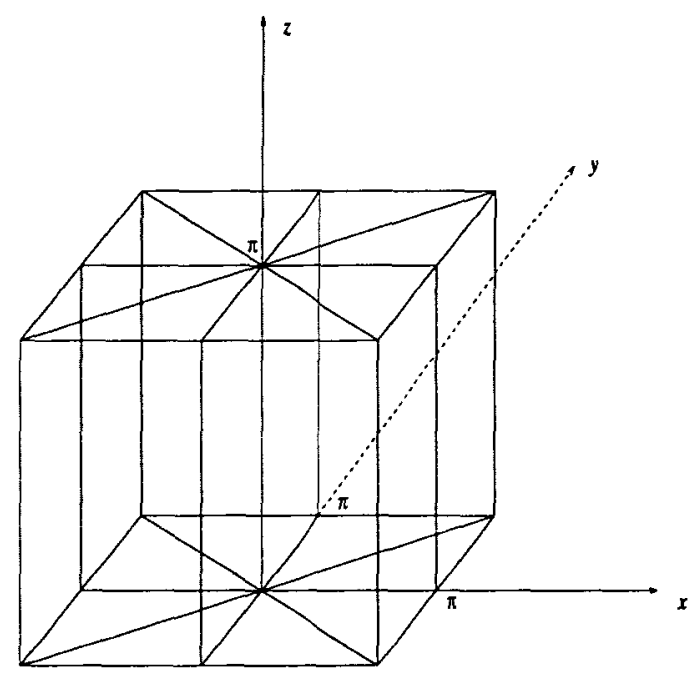

Figure 3.1. The fundamental cubical cell of the Arter flow.

Note that $(1.1)_{b=0}$ is a generalised Hamiltonian system, since (3.1)

$$
\begin{aligned}
\left(\begin{array}{l}
\frac{d x}{d t} \\
\frac{d y}{d t} \\
\frac{d z}{d t}
\end{array}\right) & =J(x, y, z) \nabla \tilde{H}(x, y, z) \\
& =\left(\begin{array}{ccc}
-\sin x \sin y \cos z & \sin x \sin y \cos z & -\sin x \cos y \sin z \\
\sin x \cos y \sin z & -\cos x \sin y \sin z & \cos x \sin y \sin z \\
0 & 0
\end{array}\right)\left(\begin{array}{c}
\frac{\cos x}{\sin x} \\
-\frac{\cos y}{\sin y} \\
0
\end{array}\right)
\end{aligned}
$$

where the Hamiltonian and Casimir functions are respectively (see $[\mathbf{2}, \mathbf{6}]$ )

$$
\widetilde{H}(x, y, z)=\log \left(\frac{\sin x}{\sin y}\right)=h^{\star},
$$


and

$$
\widetilde{C}(x, y, z)=\sin x \sin y \sin z=c^{\star} .
$$

The system (3.1) (that is, $(1.1)_{b=0}$ ) has a singular straight line $x=\pi / 2, y=\pi / 2$. By using the transformation $(\cos x, \cos y, \cos z) \rightarrow(u, v, w)$, the system $(1.1)_{b}$ can be put into the form

$$
\begin{aligned}
& \frac{d u}{d t}=\left(1-u^{2}\right) v w-2 b u\left(1-u^{2}\right)\left(2 w^{2}-1\right) \\
& \frac{d v}{d t}=\left(1-v^{2}\right) u w-2 b v\left(1-v^{2}\right)\left(2 w^{2}-1\right) \\
& \frac{d w}{d t}=-2\left(1-w^{2}\right) u v+4 b w\left(1-w^{2}\right)\left(u^{2}+v^{2}-1\right),
\end{aligned}
$$

which has Hamiltonian and Casimir functions

$$
H(u, v, w)=-\log \left(\frac{1-u^{2}}{1-v^{2}}\right)=2 h^{\star}
$$

and

$$
C(u, v, w)=\left(1-u^{2}\right)\left(1-v^{2}\right)\left(1-w^{2}\right)=c
$$

respectively, where $c=\left(c^{\star}\right)^{2}$ and we write $h=\exp \left(-2 h^{\star}\right)$. For each fixed $c=c_{0}$, $0<c_{0}<1$, on the symplectic leaf $C(u, v, w)=c_{0},(3.4)_{b}$ can be reduced to the following slowly varying system:

$$
\begin{aligned}
\frac{d u}{d t}= & w\left(1-u^{2}\right)\left(1-\frac{c_{0}}{\left(1-u^{2}\right)\left(1-w^{2}\right)}\right)^{1 / 2}-2 b u\left(1-u^{2}\right)\left(2 w^{2}-1\right) \\
\frac{d w}{d t}= & -2 u\left(1-w^{2}\right)\left(1-\frac{c_{0}}{\left(1-u^{2}\right)\left(1-w^{2}\right)}\right)^{1 / 2} \\
& +4 b w\left(1-w^{2}\right)\left(u^{2}-\frac{c_{0}}{\left(1-u^{2}\right)\left(1-w^{2}\right)}\right) \\
\frac{d c}{d t}= & 4 b c_{0}\left(2 w^{2}-u^{2}-1+\frac{c_{0}}{\left(1-u^{2}\right)\left(1-w^{2}\right)}\right),
\end{aligned}
$$

where $b$ is sufficiently small. From $(3.4)_{b}$, we know that $u= \pm 1, v= \pm 1$ and $w= \pm 1$ are invariant planes of the vector field so we only need to consider the case where $0 \leqslant|u|<1,0 \leqslant|v|<1$ and $0 \leqslant|w|<1$. We now consider the unperturbed system $(3.7)_{b=0}$ on the unit square $\left\{(u, w) \in \mathbb{R}^{2}: 0 \leqslant|u|<1,0 \leqslant|w|<1\right\}$ of the $(u, w)$-plane. The origin $(0,0)$ is a centre. The curve defined by the algebraic equation 
$\left(1-u^{2}\right)\left(1-w^{2}\right)=c_{0}, 0<c_{0}<1$, is a singular curve of equilibria of $(3.7)_{b=0}$, but when $b \neq 0$, this curve will disappear. There are two families of periodic orbits of $(3.7)_{b=0}$ given by the level curves

$$
\left(1-u^{2}\right)^{2}\left(1-w^{2}\right)=c_{0} h, \quad 0<h<c_{0} \quad \text { or } \quad 1<h<\frac{1}{c_{0}}
$$

for fixed $c_{0}$, and with the following parametric representation:

$$
\begin{aligned}
& u\left(t, k_{i}\right)= \pm\left\{\begin{array}{l}
{\left[1+\sqrt{c_{0} h}-\frac{h+\sqrt{c_{0} h}}{1-A_{1}^{2} \operatorname{sn}^{2}\left(\Omega_{1} t, k_{1}\right)}\right]^{1 / 2}, \quad 0<h<c_{0}} \\
{\left[1+\sqrt{c_{0} h}-\frac{2 \sqrt{c_{0} h}}{1-A_{2}^{2} \operatorname{sn}^{2}\left(\Omega_{2} t, k_{2}\right)}\right]^{1 / 2}, \quad 1<h<\frac{1}{c_{0}}}
\end{array}\right. \\
& w\left(t, k_{i}\right)= \pm\left[1-\frac{c_{0} h}{\left(1-u^{2}\left(t, k_{i}\right)\right)^{2}}\right]^{1 / 2},
\end{aligned}
$$

where

$$
\begin{gathered}
A_{1}^{2}=\frac{1}{2}\left(1-\sqrt{\frac{h}{c_{0}}}\right), \quad A_{2}^{2}=\frac{1-\sqrt{c_{0} h}}{1+\sqrt{c_{0} h}} \\
\Omega_{1}^{2}=2(1-h) \sqrt{\frac{c_{0}}{h}}, \quad \Omega_{2}^{2}=\left(1-\sqrt{\frac{c_{0}}{h}}\right)\left(1+\sqrt{c_{0} h}\right) \\
k_{1}^{2}=\frac{\left(\sqrt{c_{0}}-\sqrt{h}\right)\left(1+\sqrt{c_{0} h}\right)}{2 \sqrt{c_{0}}(1-h)}, \quad k_{2}^{2}=\frac{\left(1-\sqrt{c_{0} h}\right)\left(\sqrt{h}+\sqrt{c_{0}}\right)}{\left(1+\sqrt{c_{0} h}\right)\left(\sqrt{h}-\sqrt{c_{0}}\right)}
\end{gathered}
$$

in which $\operatorname{sn}(\cdot, k)$ is a Jacobi elliptic function with elliptic modulus $k$ (see [4]). The periods of the periodic orbits of (3.9) are

$$
T_{i}=\frac{2 K\left(k_{i}\right)}{\Omega_{i}}, \quad i=1,2,
$$

where $K(k)$ is the complete elliptic integral of the first kind (see [4]). We note that $h \rightarrow 1 / c_{0}$ implies that $k_{2} \rightarrow 0$, and the orbits defined by (3.9) contract to the origin $(0,0) ; h \rightarrow 0$ implies that $k_{1} \rightarrow 1 / \sqrt{2}$, and the orbits expand to the unit square. The periods $T_{i}(h)$ are monotonically varying with respect to $h, 0<h<c_{0}$ or $1<h<1 / c_{0}$. We note that for $c_{0} \leqslant h \leqslant 1$, points on the orbits of $(3.7)_{b=0}$ do satisfy the equation $\left(1-u^{2}\right)^{2}\left(1-w^{2}\right)=c_{0} h$; however, these Cartesian curves intersect the singular curve $\left(1-u^{2}\right)\left(1-w^{2}\right)=c_{0}$ as shown in Figure 3.2 and so the orbits are not periodic. 


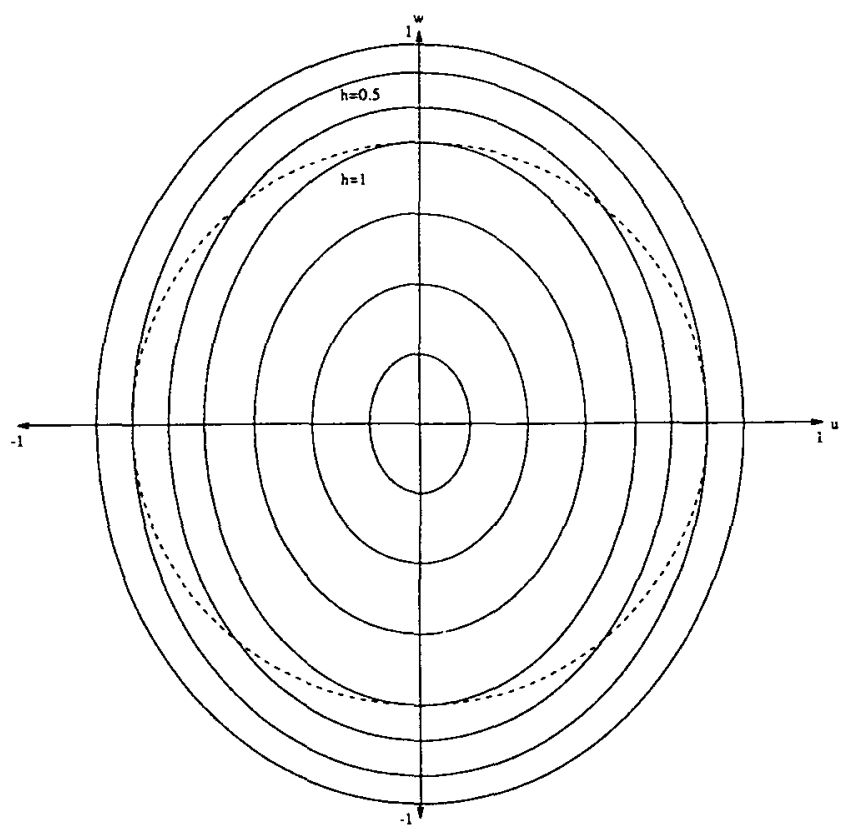

Figure 3.2. The $(u, w)$-plane of the system $(3.7)_{b=0}$ for $c_{0}=$ $1 / 2$. The dashed line is the singular curve.

On the basis of Theorem 2.3 , by using the relations

$$
w^{2}=1-\frac{c_{0} h}{\left(1-u^{2}\right)^{2}}, \quad v^{2}=1-\frac{1}{h}+\frac{1}{h} u^{2}
$$

we obtain the components $M_{1}^{i}$ and $M_{3}^{i}$ of the subharmonic Melnikov vector of (3.4) as follows:

$$
\begin{aligned}
M_{1}^{i}\left(c_{0}, h\right) & =\frac{2 T_{i}}{\pi} \int_{0}^{T_{i}}\left(v^{2}-u^{2}\right)\left(2 w^{2}-1\right) d t \\
& =\frac{2 T_{i}}{\pi}\left[2 c_{0}(1-h) \int_{0}^{T_{i}} \frac{d t}{1-u^{2}}-\frac{1-h}{h} \int_{0}^{T_{i}}\left(1-u^{2}\right) d t\right] \\
& =\frac{2 T_{i}}{\pi h}\left[2 c_{0} h(1-h) I_{-1}^{i}-(1-h) I_{1}^{i}\right]
\end{aligned}
$$


and

$$
\begin{aligned}
M_{3}^{i}\left(c_{0}, h\right) & =4 c_{0} \int_{0}^{T_{i}}\left(2 w^{2}-u^{2}-v^{2}\right) d t \\
& =4 c_{0}\left[\left(1+\frac{1}{h}\right) \int_{0}^{T_{i}}\left(1-u^{2}\right) d t-2 c_{0} h \int_{0}^{T_{i}} \frac{d t}{\left(1-u^{2}\right)^{2}}\right] \\
& =4 c_{0}\left[\left(1+\frac{1}{h}\right) I_{1}^{i}-2 c_{0} h I_{-2}^{i}\right]
\end{aligned}
$$

where

$$
I_{n}^{i}=\int_{0}^{T_{i}}\left(1-u^{2}\right)^{n} d t, \quad n=-2,-1,1 .
$$

Letting $M_{3}^{i}\left(c_{0}, h\right)=0$, we have

$$
I_{1}^{i}=\frac{2 c_{0} h^{2}}{1+h} I_{-2}^{i}
$$

and hence

$$
c_{0}=\frac{1+h}{2 h^{2}} \frac{I_{1}^{i}}{I_{-2}^{i}} .
$$

By using (3.13) and letting $M_{1}^{i}\left(c_{0}, h\right)=0$, we get

$$
I_{-1}^{i}=\frac{h}{1+h} I_{-2}^{i}
$$

Therefore, if there exists a number $h \in\left(0, c_{0}\right)$ for $i=1$ or $h \in\left(1,1 / c_{0}\right)$ for $i=2$ such that (3.13) and (3.14) hold, then on the symplectic leaf $C(u, v, w)=c_{0}$, the system $(3.4)_{b}$ has a periodic solution provided that the condition $\frac{\partial\left(M_{1}^{i}, M_{3}^{i}\right)}{\partial\left(c_{0}, h\right)} \neq 0$ is satisfied, and $b$ is sufficiently small. From Theorem 2.3 , we have the following conclusion:

ThEOREM 3.1. If there exists $h \in\left(0, c_{0}\right)$ for $i=1$ or $h \in\left(1,\left(1 / c_{0}\right)\right)$ for $i=2$ such that $I_{-1}^{i} / I_{-2}^{i}=h /(1+h), c_{0}=\left((1+h) /\left(2 h^{2}\right)\right)\left(I_{1}^{i} / I_{-2}^{i}\right)$ and $\frac{\partial\left(M_{1}^{i}\left(c_{0}, h\right), M_{3}^{i}\left(c_{0}, h\right)\right)}{\partial\left(c_{0}, h\right)} \neq 0$, then for sufficiently small $b$, the system $(3.4)_{b}$ has a periodic solution which lies on the symplectic leaf $C(u, v, w)=c_{0}$.

\section{The StRetch-TWIST-FOLD FLOW}

In this section, we consider the STF flow (1.3) $\alpha$, treating $\alpha$ and $\beta$ as parameters. In the case $\alpha \gg 1,(1.3)_{\alpha}$ can be rewritten in the form $\mathbf{v}=\mathbf{v}_{2}(x, y, z)+\varepsilon \mathbf{v}_{1}(x, y, z)$ 
where $0 \leqslant \varepsilon \ll 1$, that is,

$$
\left(\begin{array}{l}
\frac{d x}{d t} \\
\frac{d y}{d t} \\
\frac{d z}{d t}
\end{array}\right)=\left(\begin{array}{c}
z \\
0 \\
-x
\end{array}\right)+\varepsilon\left(\begin{array}{c}
-8 x y \\
11 x^{2}+3 y^{2}+z^{2}+\beta x z-3 \\
2 y z-\beta x y
\end{array}\right) .
$$

This is a perturbed generalised Hamiltonian system with Hamiltonian and Casimir functions respectively given by (see [3])

$$
H(x, y, z)=\frac{1}{2}\left(x^{2}+z^{2}\right)=h
$$

and

$$
C(x, y, z)=y .
$$

Hence, for each $C(x, y, z)=c_{0} \in \mathbb{R}$, we only need investigate the reduced system:

$$
\begin{aligned}
& \frac{d x}{d t}=z-8 \varepsilon c_{0} x \\
& \frac{d z}{d t}=-x+\varepsilon c_{0}(2 z-\beta x) \\
& \frac{d y}{d t}=\varepsilon\left(11 x^{2}+3 c_{0}^{2}+z^{2}+\beta x z-3\right) .
\end{aligned}
$$

It is easy to see that the unperturbed system (4.4) $\varepsilon_{\varepsilon=0}$ has a continuous family of periodic orbits denoted by

$$
x(t)=-\sqrt{2 h} \cos t, \quad z(t)=\sqrt{2 h} \sin t,
$$

where $h \in(0, \infty)$. Using (4.5) to compute the components $M_{1}$ and $M_{3}$ of the subharmonic Melnikov vector, we have (putting $\beta=1$ )

$$
\begin{aligned}
M_{1}\left(c_{0}, h\right) & =\int_{0}^{2 \pi}\left[c_{0} z(2 z-x)-8 c_{0} x^{2}\right] d t \\
& =\iint_{D} 6 c_{0} d x d z \\
& =-12 \pi c_{0} h,
\end{aligned}
$$

where $D$ is the region inside the periodic orbit, and

$$
\begin{aligned}
M_{3}\left(c_{0}, h\right) & =\int_{0}^{2 \pi}\left[11 x^{2}+3 c_{0}^{2}+z^{2}+x z-3\right] d t \\
& =\int_{0}^{2 \pi}\left[22 h \cos ^{2} t+2 h \sin ^{2} t-2 h \sin t \cos t+3 c_{0}^{2}-3\right] d t \\
& =2 \pi\left(12 h+3 c_{0}^{2}-3\right)
\end{aligned}
$$


From (4.6) and (4.7), we can see that if $c_{0} \neq 0$, then $M_{1}\left(c_{0}, h\right) \neq 0$. In fact, $M_{1}\left(c_{0}, h\right)=$ $M_{3}\left(c_{0}, h\right)=0$ if and only if $c_{0}=0$ and $h=1 / 4$. Since $\frac{\partial\left(M_{1}, M_{3}\right)}{\partial\left(c_{0}, h\right)} \neq 0$ at $\left(c_{0}, h\right)=$ $(0,1 / 4)$, we have the following conclusion:

THEOREM 4.1. In the plane $y=0$ of the three-dimensional phase space, near the circle $x^{2}+z^{2}=1 / 2$, there exists a periodic solution of $(4.4)_{\varepsilon}$ for sufficiently small $\varepsilon$. When $y \neq 0$, no periodic solution of $(4.4)_{\varepsilon}$ can be found.

We next discuss the case $\alpha \ll 1$. Suppose that $0 \leqslant \beta \ll 1$; the system (1.3) $\alpha$ can be rewritten in the form (replacing $\alpha$ with $\varepsilon \alpha$ and $\beta$ with $\varepsilon \beta$ where $0 \leqslant \varepsilon \ll 1$ )

$$
\begin{aligned}
& \frac{d x}{d t}=-8 x y+\varepsilon \alpha z \\
& \frac{d y}{d t}=11 x^{2}+3 y^{2}+z^{2}-3+\varepsilon \beta x z \\
& \frac{d z}{d t}=2 y z-\varepsilon x(\alpha+\beta y) .
\end{aligned}
$$

The unperturbed system $(4.8)_{\varepsilon=0}$ has the generalised Hamiltonian system form for $z \neq 0$ :

$$
\left(\begin{array}{l}
\frac{d x}{d t} \\
\frac{d y}{d t} \\
\frac{d z}{d t}
\end{array}\right)=\left(\begin{array}{ccc}
0 & -\frac{3 x^{2}+3 y^{2}+z^{2}-3}{z^{4}} & -\frac{2 y}{z^{3}} \\
\frac{3 x^{2}+3 y^{2}+z^{2}-3}{z^{4}} & 0 & \frac{2 x}{z^{3}} \\
\frac{2 y}{z^{3}} & -\frac{2 x}{z^{3}} & 0
\end{array}\right)\left(\begin{array}{c}
z^{4} \\
0 \\
4 x z^{3}
\end{array}\right)
$$

The Hamiltonian and Casimir functions are respectively (see [3])

$$
H(x, y, z)=x z^{4}=h
$$

where $h \in \mathbb{R}$, and

$$
C(x, y, z)=\frac{x^{2}+y^{2}+z^{2}-1}{z^{3}}=c
$$

where $c \in \mathbb{R}$. On the symplectic leaf $C(x, y, z)=c_{0},(4.8)_{\varepsilon}$ can be reduced to

$$
\begin{aligned}
& \frac{d z}{d t}=2 y z-\varepsilon(\alpha+\beta y)\left(c_{0} z^{3}-y^{2}-z^{2}+1\right)^{1 / 2} \\
& \frac{d y}{d t}=11 c_{0} z^{3}-8 y^{2}-10 z^{2}+8+\varepsilon \beta z\left(c_{0} z^{3}-y^{2}-z^{2}+1\right)^{1 / 2} \\
& \frac{d c}{d t}=3 \varepsilon c_{0}(\alpha+\beta y)\left(c_{0} z^{3}-y^{2}-z^{2}+1\right)^{1 / 2} z^{-1}
\end{aligned}
$$


The reduced unperturbed system (4.12) $)_{\varepsilon=0}$ is integrable. Let $f(z)=11 c_{0} z^{3}-10 z^{2}+$ $8, z \in \mathbb{R}$. It is easy to see that if $c_{0}>0$ and $f\left((20) /\left(33 c_{0}\right)\right)=-(10 / 3)\left(20 / 33 c_{0}\right)^{2}+8 \leqslant 0$ (that is, $\left.c_{0} \leqslant(10 \sqrt{5}) /(33 \sqrt{3})\right)$, then $f$ has one negative real root and two positive real roots denoted by $r_{1}<0<r_{2} \leqslant r_{3}$; if $f\left((20) /\left(33 c_{0}\right)\right)>0, f$ only has one negative real root $r_{1}$. By a simple analysis, we know that in the phase plane $(z, y)$, the phase portraits of $(4.12)_{\varepsilon=0}$ can be shown as in Figure 4.1.
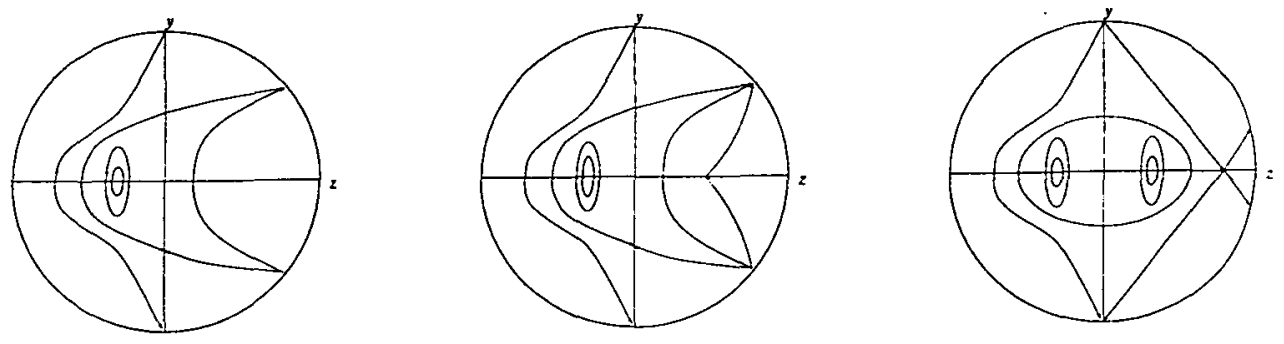
(a) $f\left(\frac{20}{33 c_{0}}\right)>0$
(b) $f\left(\frac{20}{33 c_{0}}\right)=0$
(c) $f\left(\frac{20}{33 c_{0}}\right)<0$

Figure 4.1. The phase portraits of $(4.12)_{\varepsilon=0}$.

In cases (a) and (b) of Figure 4.1, there is a family of periodic orbits of $(4.12)_{\varepsilon=0}$ corresponding to the Hamiltonian function

$$
z^{4} \sqrt{c_{0} z^{3}-y^{2}-z^{2}+1}=h, \quad h \in\left(0, r_{1}^{4}\left(c_{0} r_{1}^{3}-r_{1}^{2}+1\right)^{\frac{1}{2}}\right) .
$$

For case (c) of Figure 4.1, there are two families of periodic solutions of $(4.12)_{\varepsilon=0}$, respectively corresponding to $h \in\left(0, r_{i}^{4}\left(c_{0} r_{i}^{3}-r_{i}^{2}+1\right)^{1 / 2}\right), i=1,2$. Along each closed orbit of (4.12) $)_{\varepsilon=0}$, we compute the components $M_{1}$ and $M_{3}$ of the subharmonic Melnikov vector. We have

$$
\begin{aligned}
M_{1}\left(\alpha, \beta, h, c_{0}\right) & =\int_{0}^{T}\left[\left(11 c_{0} z^{3}-8 y^{2}-10 z^{2}+8\right)(\alpha+\beta y) x+2 \beta x y z\right] z^{7} d t \\
& =\iint_{D}\left[\frac{\partial}{\partial y}\left(\beta h z^{3}\right)-\frac{\partial}{\partial z}\left((\alpha+\beta y) h z^{3}\right)\right] d y d z \\
& =-3 h \iint_{D}(\alpha+\beta y) z^{2} d y d z \\
& =-3 \alpha h \iint_{D} z^{2} d y d z
\end{aligned}
$$


where $D$ is the region inside the periodic orbit. Also,

$$
\begin{aligned}
M_{3}\left(\alpha, \beta, h, c_{0}\right) & =3 c_{0} \int_{0}^{T}(\alpha+\beta y)\left(c_{0} z^{3}-y^{2}-z^{2}+1\right)^{1 / 2} z^{-1} d t \\
& =3 c_{0} h \int_{0}^{T}(\alpha+\beta y) z^{-5} d t \\
& =3 c_{0} h \int_{z_{\min }}^{z_{\max }}(\alpha+\beta y) \frac{1}{2 y z^{6}} d z \\
& =\frac{3}{2} c_{0} h\left[\alpha \int_{z_{\min }}^{z_{\max }} \frac{d z}{z^{6}\left(c_{0} z^{3}-z^{2}-\frac{h^{2}}{z^{8}}+1\right)^{1 / 2}}+\beta \int_{z_{\min }}^{z_{\max }} \frac{d z}{z^{6}}\right]
\end{aligned}
$$

where $z_{\min }$ and $z_{\max }$ are the two intersection points of the periodic orbit with the $z$ axis. From (4.13), we know that if $\alpha h \neq 0$ then $M_{1}\left(\alpha, \beta, h, c_{0}\right) \neq 0$, since the integral $\iint_{D} z^{2} d y d z \neq 0$, so that we have the following result:

THEOREM 4.2. If $\alpha h \neq 0$, then on each symplectic leaf $C(x, y, z)=c_{0}$, no periodic solution of $(4.8)_{\varepsilon}$ can be found.

If $h=0$, this means that $z=0$ or $x=0$ from (4.10). On the invariant planes $z=0$ and $x=0$, the unperturbed system $(4.8)_{\varepsilon=0}$ has two families of periodic orbits. For $(4.8)_{\varepsilon}$ with $\varepsilon \neq 0$, do these periodic orbits persist? This is a problem for future study.

\section{Modulated travelling Waves in thermal CONVECtion}

Consider the system

$$
\begin{aligned}
& \frac{d s_{1}}{d t}=s_{1} s_{2} \cos \phi+\varepsilon s_{1}\left(\nu_{1}+e_{11} s_{1}^{2}+e_{12} s_{2}^{2}\right) \\
& \frac{d s_{2}}{d t}=-s_{1}^{2} \cos \phi+\varepsilon s_{2}\left(\nu_{2}+e_{21} s_{1}^{2}+e_{22} s_{2}^{2}\right) \\
& \frac{d \phi}{d t}=-\left(2 s_{2}-\frac{s_{1}^{2}}{s_{2}}\right) \sin \phi .
\end{aligned}
$$

This system has been studied by Armbruster, Guckenheimer and Holmes [1]. In this section, we shall show that using the theory of perturbed generalised Hamiltonian systems, we can obtain a clearer understanding for the existence of modulated travelling waves. The system $(5.1)_{\varepsilon=0}$ has the generalised Hamiltonian form

$$
\left(\begin{array}{c}
\frac{d s_{1}}{d t} \\
\frac{d s_{2}}{d t} \\
\frac{d \phi}{d t}
\end{array}\right)=\left(\begin{array}{ccc}
0 & 0 & \frac{1}{s_{1}} \\
0 & 0 & -\frac{1}{s_{2}} \\
-\frac{1}{s_{1}} & \frac{1}{s_{2}} & 0
\end{array}\right)\left(\begin{array}{c}
2 s_{1} s_{2} \sin \phi \\
s_{1}^{2} \sin \phi \\
s_{1}^{2} s_{2} \cos \phi
\end{array}\right)
$$


where the Hamiltonian and Casimir functions are respectively (see [1])

$$
H\left(s_{1}, s_{2}, \phi\right)=s_{1}^{2} s_{2} \sin \phi=h,
$$

and

$$
C\left(s_{1}, s_{2}, \phi\right)=s_{1}^{2}+s_{2}^{2}=c .
$$

For a fixed $c=c_{0}$, let $s_{1}=\sqrt{c_{0}} \cos \theta, s_{2}=\sqrt{c_{0}} \sin \theta$. The system $(5.1)_{\varepsilon}$ can be reduced to the following new system on the symplectic cylinder $C\left(s_{1}, s_{2}, \phi\right)=c_{0}$ :

$$
\begin{aligned}
& \frac{d \theta}{d t}=-\sqrt{c_{0}} \cos \theta \cos \phi-\varepsilon \cos \theta \sin \theta\left[\nu_{1}-\nu_{2}\right. \\
&\left.+\left(e_{11}-e_{21}\right) c_{0} \cos ^{2} \theta+\left(e_{12}-e_{22}\right) c_{0} \sin ^{2} \theta\right] \\
& \frac{d \phi}{d t}=- \sqrt{c_{0}}\left(2 \sin \theta-\frac{\cos ^{2} \theta}{\sin \theta}\right) \sin \theta \\
& \frac{d c}{d t}=2 \varepsilon c_{0}\left[\cos ^{2} \theta\left(\nu_{1}+e_{11} c_{0} \cos ^{2} \theta+e_{12} c_{0} \sin ^{2} \theta\right)\right. \\
&\left.+\sin ^{2} \theta\left(\nu_{2}+e_{21} c_{0} \cos ^{2} \theta+e_{22} c_{0} \sin ^{2} \theta\right)\right]
\end{aligned}
$$

When $\theta \in[0,(\pi / 2)]$ and $\phi \in[0, \pi]$, that is, in the rectangle $[0,(\pi / 2)] \times[0, \pi]$ of the phase plane $(\theta, \phi)$, the unperturbed system $(5.4)_{\epsilon=0}$ has a centre at $(\arctan (1 / \sqrt{2}), \pi / 2)$ surrounded by a continuous family of periodic orbits. Write $A^{2}=\left(h^{2} / c_{0}^{3}\right)$, and observe that $A^{2} \leqslant 4 / 27$. Let $\alpha, \beta$ and $\gamma$ be the three roots of the equation $1-8 A+u-u^{2}-u^{3}=0$. We have the following parametric representation of the periodic orbits of $(5.4)_{\varepsilon=0}$ :

$$
\begin{aligned}
& \theta(t, k)=\frac{1}{2} \arccos \left[\alpha-(\alpha-\beta) \operatorname{sn}^{2}(\Omega t, k)\right], \\
& \phi(t, k)=\arccos \left[-\frac{\frac{d \theta}{d t}(t, k)}{\sqrt{c_{0}} \cos \theta(t, k)}\right],
\end{aligned}
$$

where $\Omega=\sqrt{(\alpha-\gamma) /\left(2 c_{0}\right)}, k=\sqrt{(\alpha-\beta) /(\alpha-\gamma)}$. The period of the orbit (5.5) is $T=(2 K(k)) / \Omega$. To investigate the existence of periodic orbits of $(5.4)_{\varepsilon}$, we consider the components $M_{1}$ and $M_{3}$ of the subharmonic Melnikov vector:

$$
\begin{aligned}
& M_{1}\left(\nu_{1}, \nu_{2}, e_{11}, e_{12}, e_{21}, e_{22}, c_{0}, h\right) \\
& =\sqrt{c_{0}} \int_{0}^{T}\left(\cos ^{2} \theta-\sin ^{2} \theta\right)\left[\nu_{1}-\nu_{2}+c_{0}\left(e_{11}-e_{21}\right) \cos ^{2} \theta+c_{0}\left(e_{12}-e_{22}\right) \sin ^{2} \theta\right] d t
\end{aligned}
$$


and

$$
\begin{aligned}
M_{3}\left(\nu_{1}, \nu_{2}, e_{11}, e_{12}, e_{21}, e_{22}, c_{0}, h\right) & =\sqrt{c_{0}} \int_{0}^{T}\left\{\nu_{1} \cos ^{2} \theta+\nu_{2} \sin ^{2} \theta\right. \\
& \left.+\sqrt{c_{0}}\left[e_{11} \cos ^{4} \theta+\left(e_{12}+e_{21}\right) \cos ^{2} \theta \sin ^{2} \theta+e_{22} \sin ^{4} \theta\right]\right\} d t
\end{aligned}
$$

To calculate these integrals, we note that

$$
\left[\frac{d \theta}{d t}\right]^{2}=c_{0} \cos ^{2} \theta\left(1-\frac{A^{2}}{\cos ^{4} \theta \sin ^{2} \theta}\right), \quad\left[\frac{d}{d t}\left(\cos ^{2} \theta\right)\right]^{2}=4 c_{0}\left[\cos ^{4} \theta-\cos ^{6} \theta-A^{2}\right]
$$

Letting $\tau=2 \sqrt{c_{0}} t$ and $m=\cos ^{2} \theta$, and defining $m_{1}$ and $m_{2}\left(m_{1}<m_{2}\right)$ as the two positive roots of the equation $m^{2}-m^{3}-A^{2}=0$, then we have the period

$$
T^{\star}(A)=2 \int_{m_{1}}^{m_{2}} \frac{d m}{\sqrt{m^{2}-m^{3}-A^{2}}}=I_{0},
$$

which is with respect to the timescale $\tau$. Denote

$$
I_{1}=\int_{0}^{T^{\star}} \cos ^{2} \theta(\tau) d \tau=2 \int_{m_{1}}^{m_{2}} \frac{m}{\sqrt{m^{2}-m^{3}-A^{2}}} d m, \quad I_{i}=2 \int_{m_{1}}^{m_{2}} \frac{m^{i}}{\sqrt{m^{2}-m^{3}-A^{2}}} d m .
$$

Taking into account that

$$
2 I_{1}-3 I_{2}=2 \int_{m_{1}}^{m_{2}} \frac{2 m-3 m^{2}}{\sqrt{m^{2}-m^{3}-A^{2}}} d m=4\left[\sqrt{m^{2}-m^{3}-A^{2}}\right]_{m_{1}}^{m_{2}}=0
$$

we have

$$
\begin{gathered}
\int_{0}^{T^{\star}} \cos ^{4} \theta d \tau=I_{2}=\frac{2}{3} I_{1}, \quad \int_{0}^{T^{\star}} \sin ^{2} \theta d \tau=I_{0}-I_{1} \\
\int_{0}^{T^{\star}} \sin ^{2} \theta \cos ^{2} \theta d \tau=I_{1}-I_{2}=\frac{1}{3} I_{1}, \quad \int_{0}^{T^{\star}} \sin ^{4} \theta d \tau=I_{0}-2 I_{1}+I_{2}=I_{0}-\frac{4}{3} I_{1} .
\end{gathered}
$$

Thus, we obtain

$$
M_{1}\left(\nu_{1}, \nu_{2}, e_{11}, e_{12}, e_{21}, e_{22}, c_{0}, h\right)=\sqrt{c_{0}}\left[3 I_{1}-2 I_{0}\right]\left[\nu_{1}-\nu_{2}+c_{0}\left(e_{12}-e_{22}\right)\right]
$$

and

$$
\begin{aligned}
M_{3}\left(\nu_{1}, \nu_{2}, e_{11}, e_{12}, e_{21}, e_{22}, c_{0}, h\right) & \\
= & \sqrt{c_{0}}\left\{\nu_{1} I_{1}+\nu_{2}\left(I_{0}-I_{1}\right)+\frac{1}{3} \sqrt{c_{0}}\left[2 e_{11} I_{1}+\left(e_{12}+e_{21}\right) I_{1}+e_{22}\left(3 I_{0}-4 I_{1}\right)\right]\right\} \\
& =\sqrt{c_{0}}\left\{\left(\nu_{1}-\nu_{2}\right) I_{1}+\nu_{2} I_{0}+\frac{1}{3} \sqrt{c_{0}}\left[\left(2 e_{11}+e_{12}+e_{21}-4 e_{22}\right) I_{1}+3 e_{22} I_{0}\right]\right\}
\end{aligned}
$$


Next, we discuss the zeros of the components $M_{1}$ and $M_{3}$ of the Melnikov vector. From (5.6), we see that $M_{1}\left(\nu_{1}, \nu_{2}, e_{11}, e_{12}, e_{21}, e_{22} ; c_{0}, h\right)=0$ if and only if

$$
I_{1}=\frac{2}{3} I_{0} \quad \text { or } \quad c_{0}=\frac{\nu_{2}-\nu_{1}}{e_{12}-e_{22}} .
$$

$I_{1}=(2 / 3) I_{0}$ corresponds to the relation $h^{2}=(4 / 27) c_{0}^{3}$, that is, the Hamiltonian value at the centre equilibrium. The second relation of (5.8) implies that for the given parameter set $\left(\nu_{1}, \nu_{2}, e_{12}, e_{22}\right)$, there exists a unique $c_{0}$ such that on the symplectic leaf $C(x, y, z)=c_{0}$, the system $(5.4)_{\varepsilon}$ may have a periodic solution. From (5.7), we know that taking $c_{0}=\left(\nu_{2}-\nu_{1}\right) /\left(e_{12}-e_{22}\right)$ under the condition

$$
\left(\nu_{2}-\nu_{1}\right) I_{1}-\nu_{2} I_{0}=(1 / 3) \sqrt{\frac{\nu_{2}-\nu_{1}}{e_{12}-e_{22}}}\left[\left(2 e_{11}+e_{12}+e_{21}-4 e_{22}\right) I_{1}+3 e_{22} I_{0}\right],
$$

we have $M_{3}\left(\nu_{1}, \nu_{2}, e_{11}, e_{12}, e_{21}, e_{22}, c_{0}, h\right)=0$. For every given value of $h$ such that $0<h^{2}<(4 / 27) c_{0}^{3},(5.9)$ determines a curve in the parameter plane $\left(\nu_{1}, \nu_{2}\right)$ such that there exists a periodic solution of $(5.4)_{\varepsilon}$. Of course, we need to check the condition $\frac{\partial\left(M_{1}, M_{3}\right)}{\partial\left(c_{0}, h\right)} \neq 0$. This is clear from the result of Armbruster, Guckenheimer and Holmes [1] so we omit it. The following conclusion coincides with [1, Theorem 4.1]:

THEOREM 5.1. For a given parameter group $\left(\nu_{1}, \nu_{2}, e_{11}, e_{12}, e_{21}, e_{22}\right)$, there is a domain in the $\left(\nu_{1}, \nu_{2}\right)$-plane such that on the symplectic leaf $s_{1}^{2}+s_{2}^{2}=\left(\nu_{2}-\nu_{1}\right) /\left(e_{12}-e_{22}\right)$, the system $(5.1)_{\epsilon}$ has a unique periodic orbit, which corresponds to the modulated travelling wave solution in thermal convection.

\section{REFERENCES}

[1] D. Armbruster, J. Guckenheimer and P. Holmes, 'Heteroclinic cycles and modulated travelling waves in systems with $\mathrm{O}(2)$ symmetry', Phys. D 29 (1988), 257-282.

[2] W. Arter, 'Ergodic stream-lines in steady convection', Phys. Lett. A 97 (1983), 171-174.

[3] K. Bajer and H.K. Moffatt, 'On a class of steady confined Stokes flows with chaotic streamlines', J. Fluid Mech. 212 (1990), 337-363.

[4] P.F. Byrd and M.D. Friedman, Handbook of elliptic integrals for engineers and scientists (Springer-Verlag, Berlin, 1971).

[5] S. Chandrasekhar, Hydrodynamic and hydromagnetic stability (Clarendon, Oxford, 1961).

[6] D.D. Holm and Y. Kimura, 'Zero-helicity Lagrangian kinematics of three-dimensional advection', Phys. Fluids A 3 (1991), 1033-1038.

[7] C.A. Jones and M.R.E. Proctor, 'Strong spatial resonance and travelling waves in Bénard convection', Phys. Lett. A 121 (1987), 224-228.

[8] J. Li, X. Zhao and Z. Liu, Theory and application of generalized Hamiltonian systems, (in Chinese) (Science Press, Beijing, 1994). 
[9] V.K. Melnikov, 'On the stability of the center for time periodic perturbations', Trans. Moscow Math. Soc. 12 (1963), 1-57.

[10] H.K. Moffatt and M.R.E. Proctor, 'Topological constraints associated with fast dynamo action', J. Fluid Mech. 154 (1985), 493-507.

[11] P.J. Olver, Applications of Lie groups to differential equations, (Second edition) (SpringerVerlag, New York, 1993).

[12] M.R.E. Proctor and C.A. Jones, 'The interaction of two spatially resonant patterns in thermal convection. Part 1. Exact 1:2 resonance', J. Fluid Mech. 188 (1988), 301-335.

[13] S. Smale, 'Dynamics retrospective: Great problems, attempts that failed', Phys. D 51 (1991), 267-273.

[14] A. Weinstein, 'The local structure of Poisson manifolds', J. Differential Geom. 18 (1983), 523-557.

[15] S. Wiggins and P. Holmes, 'Periodic orbits in slowly varying oscillators', SIAM J. Math. Anal. 18 (1987), 592-611.

Institute of Applied Maths of Yunnan Province Department of Mathematics

Kunming University of Science \& Technology

Yunnan 650093

People's Republic of China

e-mail: jibinli@ynu.edu.cn

Department of Mathematics and Statistics

The Flinders University of South Australia

GPO Box 2100

Adelaide SA 5001

Australia

e-mail: gopal@ist.flinders.edu.au
Department of Mathematics and Statistics The Flinders University of South Australia GPO Box 2100

Adelaide SA 5001

Australia

e-mail: johnc@ist.flinders.edu.au 